\title{
Paradoxical Onset of Arrhythmic Waves from Depolarized Areas in Cardiac Tissue Due to Curvature-Dependent Instability
}

\author{
Alexander S. Teplenin, ${ }^{1}$ Hans Dierckx,${ }^{2}$ Antoine A. F. de Vries, ${ }^{1}$ Daniël A. Pijnappels, ${ }^{1, *}$ and Alexander V. Panfilov ${ }^{1,2,3, \uparrow}$ \\ ${ }^{1}$ Laboratory of Experimental Cardiology, Department of Cardiology, Heart Lung Center Leiden, \\ Leiden University Medical Center, Leiden, the Netherlands \\ ${ }^{2}$ Department of Physics and Astronomy, Ghent University, Ghent, Belgium \\ ${ }^{3}$ Ural Federal University, Ekaterinburg, Russia
}

(Received 27 October 2017; revised manuscript received 18 April 2018; published 26 June 2018)

\begin{abstract}
The generation of abnormal excitations in pathological regions of the heart is a main trigger for lethal cardiac arrhythmias. Such abnormal excitations, also called ectopic activity, often arise from areas with local tissue heterogeneity or damage accompanied by localized depolarization. Finding the conditions that lead to ectopy is important to understand the basic biophysical principles underlying arrhythmia initiation and might further refine clinical procedures. In this study, we are the first to address the question of how geometry of the abnormal region affects the onset of ectopy using a combination of experimental, in silico, and theoretical approaches. We paradoxically find that, for any studied geometry of the depolarized region in optogenetically modified monolayers of cardiac cells, primary ectopic excitation originates at areas of maximal curvature of the boundary, where the stimulating electrotonic currents are minimal. It contradicts the standard critical nucleation theory applied to nonlinear waves in reaction-diffusion systems, where a higher stimulus is expected to produce excitation more easily. Our in silico studies reveal that the nonconventional ectopic activity is caused by an oscillatory instability at the boundary of the damaged region, the occurrence of which depends on the curvature of that boundary. The onset of this instability is confirmed using the Schrödinger equation methodology proposed by Rinzel and Keener [SIAM J. Appl. Math. 43, 907 (1983)]. Overall, we show distinctively novel insight into how the geometry of a heterogeneous cardiac region determines ectopic activity, which can be used in the future to predict the conditions that can trigger cardiac arrhythmias.
\end{abstract}

DOI: 10.1103/PhysRevX.8.021077

Subject Areas: Biological Physics, Interdisciplinary Physics, Nonlinear Dynamics

\section{INTRODUCTION}

Arrhythmias are the most common cause of sudden cardiac death worldwide, accounting for an estimated 6 million deaths annually [1]. However, the underlying mechanisms are still not completely understood. From a practical point of view, it is of paramount importance to understand the mechanisms of arrhythmia initiation, since by removing the triggers, arrhythmic events can be avoided. Multiple recordings have shown that, in many cases, lethal cardiac arrhythmias are triggered by so-called ectopic beats, i.e., ill-timed electrical pulses originating from damaged tissue, which disturb normal cardiac

\footnotetext{
*D.A.Pijnappels@lumc.nl

†Alexander.Panfilov@uGent.be
}

Published by the American Physical Society under the terms of the Creative Commons Attribution 4.0 International license. Further distribution of this work must maintain attribution to the author(s) and the published article's title, journal citation, and DOI. rhythm [2,3]. Despite their practical importance, the mesoscopic biophysical mechanisms of ectopic activity remain largely unknown [4]. In a very general sense, ectopic activity occurs when a depolarized region in the heart produces depolarizing currents strong enough to initiate propagating waves.

The process of wave initiation by such depolarizing currents seems to be straightforward at first sight: larger currents or larger sources can more easily depolarize a cell, overcome the sink effect from adjacent cells, and produce a propagating ectopic wave.

This principle works perfectly to describe curvaturevelocity relationships of waves in excitable media [5]. It closely relates to the nucleation theory of phase transitions [6,7], since one also needs to form a critical volume to initiate a phase transition in a metastable phase of matter. In electrophysiology, this paradigm is called the sourcesink relationship [8].

The depolarizing current by itself may depend on many factors, including the shape of the ectopic region. The effect of the shape of injury-induced depolarized zones on ectopic 
activity has so far not been studied in depth because it was previously impossible to produce a depolarized region of a given shape due to various technical limitations $[9,10]$. Currently, this issue can be resolved by applying optogenetic techniques, which were first applied in neuroscience [11] and allow excellent spatiotemporal control of cellular properties and wave dynamics in biological excitable media $[12,13]$. Capitalizing on these advantages, we previously showed that optogenetically induced localized oxidative stress can be used to generate intentionally shaped zones with sustained depolarization and associated ectopic activity [14]. In the current work, this technique was applied to induce quasistable depolarized (QSD) zones of predefined shapes in monolayers of cardiac cells.

Remarkably, the ectopic activity was found to preferentially emerge from the sharp convex corners of QSD tissue regions. This observation defies the conventional interpretation of the source-sink concept, as in convex corners of the QSD region, the density of the depolarizing current is minimal.

In order to understand this paradoxical phenomenon, we conducted numerical simulations and reproduced the effect in physiologically detailed models and even in simple reactiondiffusion models. Below, we will link the phenomenon to the occurrence of oscillatory instability at the boundary of the QSD region and explain the onset of this instability semianalytically using the stationary Schrödinger equation.

\section{MATERIALS AND METHODS}

A more detailed description is provided in Appendix B.

\section{A. Ectopic activity caused by optogenetic production of reactive oxygen species}

A plasma membrane-targeted version of a mini-singlet oxygen generator (miniSOG) [15] was expressed in neonatal rat ventricular myocyte (NRVM) monolayers following lentiviral transduction, as reported previously [16,17]. Lentiviral vector particles were produced from shuttle plasmid pLV.hCMV-IE.miniSOG-PM.hHBVPRE, as detailed elsewhere [17]. The mode of action of miniSOG and the proviral DNA structure of the lentiviral vector are shown in Figs. S1(a) and S1(b) of the Supplemental Material [18], respectively. After establishing practically uniform transduction with miniSOG-encoding lentiviral vector particles, optical mapping was performed on eight- to ten-day-old cardiac monolayer cultures with the fluorescent voltagesensitive dye di-4-ANEPPS to observe excitation waves. A patterned projection system was used to focus $470-\mathrm{nm}$ LED light in the plane of the monolayers, in a region of maximal size $6 \times 6 \mathrm{~mm}$. After irradiation with intensity $0.31 \mathrm{~mW} / \mathrm{mm}^{2}$ for 3-6 $\mathrm{min}$, to induce reactive oxygen species (ROS) production, voltage was optically monitored. Cumulative ROS damage resulted in ultralong action potentials (APs) in the previously irradiated zone, lasting for
2-20 s. Light-induced ROS production did not change AP duration (APD) in the unexposed parts of the monolayers, which had an $\mathrm{APD}_{80}$ of $200-350 \mathrm{~ms}$. The drastic spatial difference in APD caused ectopic waves to emanate from the interface between the normal and damaged tissue regions, as shown in the two examples of paired optical traces presented in Fig. S1(d) of the Supplemental Material [18]. While, in both examples, the optical signals from electrical activity in most of the irradiated region remained almost flat with a long plateau phase, the surrounding tissue exhibited periodic APs. The ectopic beats emerged only during the plateau phase of ultralong APs, in line with our previous findings [14].

\section{B. Mathematical model for normal and QSD tissue}

A hierarchy of models of different complexity was used, including the Majumder-Korhonen model for NRVMs [19], which is the model closest to our in vitro setup. We employed the Ten Tusscher-Noble-NoblePanfilov (TNNP) model for adult human ventricular myocytes [20] to study in silico possible manifestations of the effects in human ventricular tissue. We also used the FitzHugh-Nagumo (FHN) [21] and the Aliev-Panfilov model [22], which were modified to study the generic mechanisms underlying the observed effects. A detailed description of all four models is given in Appendix B.

To identify the mechanism behind the ectopic activity observed in our in vitro experiments, a generic description of the tissue was used in order to relate the observed phenomena to the most fundamental properties of cardiac excitation, i.e., excitation of cardiomyocytes by local currents and establishment of a transient refractory state in the cells following excitation. In our view, this can best be achieved by employing low-dimensional models, which focus on these processes, rather than by relying on complex descriptions of the underlying ionic currents and $\mathrm{Ca}^{2+}$ dynamics. Simplified models often describe complex spatiotemporal phenomena without loss of essential details (see, e.g., Ref. [23]). Therefore, we used the classical cubic FHN model [21] in one (1D) or two (2D) spatial dimensions:

$$
\frac{\partial u}{\partial t}=-f(u)-v+D \Delta u, \quad \frac{\partial v}{\partial t}=\varepsilon[u-\gamma(\vec{r}) v],
$$

where $u$ is the normalized transmembrane potential, $v$ represents recovery processes, $f(u)=u(u-1)(u-a)$, $0<a<0.5$, and $\varepsilon \ll 1$. To distinguish between illuminated (i.e., oxidatively damaged) tissue exhibiting ultralong APs (i.e., quasistable depolarization) and normal (i.e., nonilluminated) tissue, we altered the slope of the second variable $\gamma(\vec{r})$ as follows. Normal tissue was modeled as a monostable system $\left(\gamma=\gamma_{\text {mono }}=1.5\right)$, as it allows an excitable regime. After excitation $(u \approx 1)$, the system returns to the resting potential $(u=0)$; see the nullclines in Fig. 3(a). Such a monostable system supports propagating waves as well. 
The tissue exhibiting quasistable depolarization, however, stays in the depolarized state much longer than the duration of a normal AP. The QSD tissue reaches this depolarized state from rest only after a depolarizing wave has passed through it. The described computational model contains a slow timescale variable, which, in the limiting case, can be adiabatically eliminated [24], and QSD tissue can, therefore, be modeled as a bistable system, which can be realized in Eq. (1) by choosing $\gamma=\gamma_{b i}>5.85$, producing stable states $u=0$ and $u=u_{3}>0$, as shown in Fig. 3(a). The other parameters of the model were taken to be $a=0.13$, $\varepsilon=0.004$, and $D=2.0$. The size of the computational domain was $1024 \times 1024$, with a central bistable square zone of size $400 \times 400$. Parameters for other shapes and models are described in Appendix B.

\section{RESULTS}

\section{A. Effect of QSD tissue geometry on ectopic beat generation in optogenetic experiments with cardiac monolayer cultures}

In the first set of experiments, we generated ultralong APs in a square region (i.e., QSD region) of $6 \times 6 \mathrm{~mm}$ by local light-induced production of ROS and studied its effect on AP propagation. Following electrical stimulation, an initial wave of excitation propagated through the tissue, inducing long-lasting depolarization inside the square. This generated secondary excitation waves (i.e., ectopic beats) at the corners of the previously illuminated area, which subsequently traveled through the medium; see Fig. 1(a). The spatiotemporal organization of electrical activity during the ectopic beats is shown in Fig. 1(b). Consistent with our previous findings, the center of the illuminated area (green trace) entered into a prolonged state of depolarization (i.e., became QSD) without significant oscillations, while the unexposed tissue produced normal APs (magenta trace). The border zone between both regions showed larger oscillations (blue trace) than the center of the illuminated area. Moving from the center to the periphery of the cell monolayer, there were gradients in the amplitude of the oscillations indicative of electrotonic effects. The observed wave emission is paradoxical, for the following reason. The square region produces depolarizing electrotonic currents that excite the normal surrounding tissue of lower membrane potential. The density of the depolarizing current obviously depends on the shape of the heterogeneity, and it will be lowest at its corners, where the ratio of depolarized to repolarized tissue is minimal. Because of this unfavorable source-sink relationship, the corners of the square are the least likely regions for impulse generation, which is expected to occur in the most concave regions of the interface, e.g., in the middle of the square's edges.

To further study how the boundary geometry affects ectopic activity, additional optogenetic experiments were
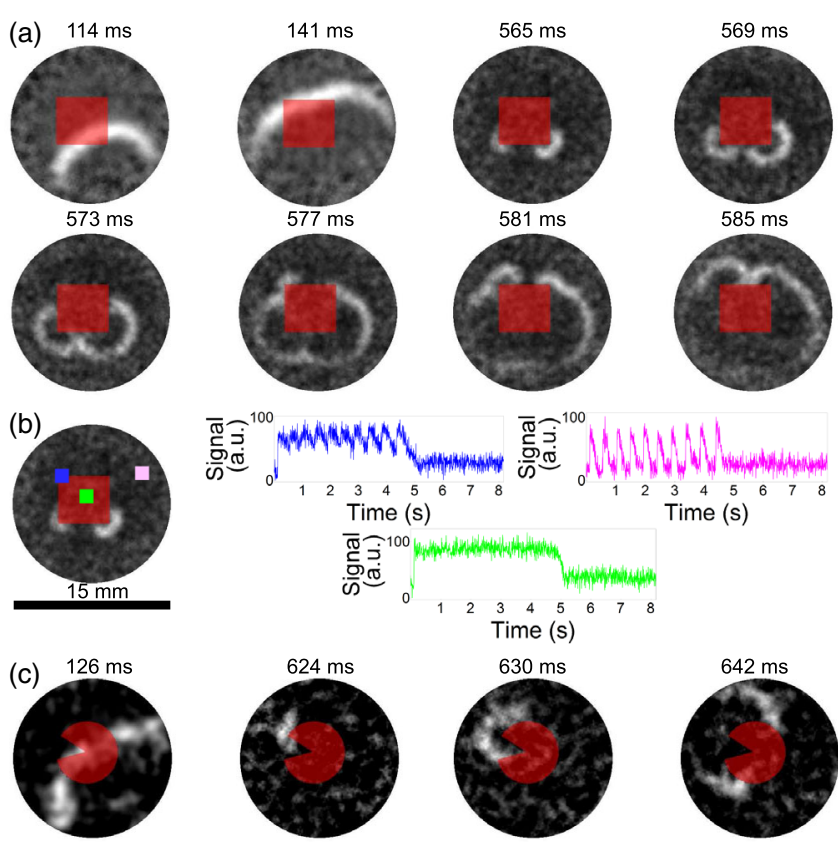

Time (s)

(d) $18 \mathrm{~ms}$
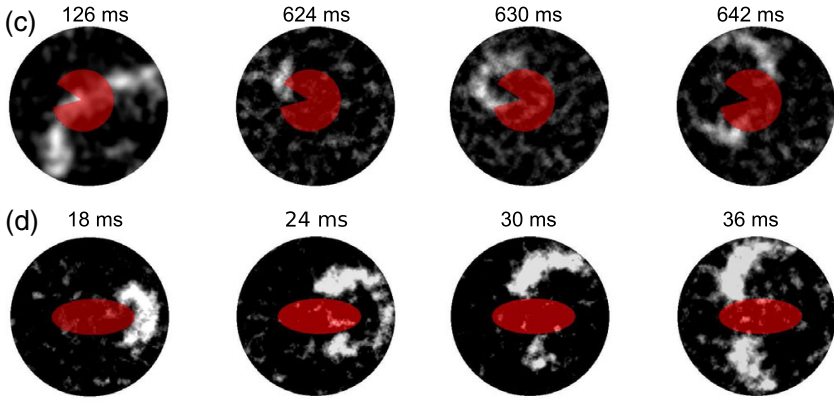

FIG. 1. Optogenetic induction of quasistable depolarized zones (red areas) leads to ectopic waves emanating from the most curved portions of the interface between tissue with ultralong and normal action potentials. (a) Time series of a single experiment showing ectopic waves originating from the corners of a square quasistable depolarized region after a first wave has passed. (b) Distribution of the oscillations amplitude during ectopic activity at the positions indicated by the green, blue, and magenta dot. (c) Generation of an ectopic wave from the "jaw tip of a Pacman." (d) Generation of an ectopic wave from the vertex of an elliptic interface. In all figures, the symmetry is broken by the first wave passing through the medium.

performed, in which areas of ultralong APD had a Pacmanlike or elliptic shape; see Figs. 1(c) and 1(d). In line with the results presented in Fig. 1(a), the first ectopic beat emerged from the "jaw tip" of the Pacman rather than from its "mouth," as shown in frames 2-4 of Fig. 1(c). A similar result was obtained for the elliptic heterogeneity, since once again the ectopic activity was generated at the sites of highest curvature (i.e., the vertices) [Fig. 1(d)]. In total, such dynamics was observed in 29 out of 36 experiments for the square-shaped heterogeneity, in 5 out of 5 cases for the Pacman-like heterogeneity, and in 3 out of 4 cases for the elliptical heterogeneity. Our in vitro experiments, thus, robustly show that, contrary to what would be expected based on classical source-sink considerations, ectopic activity can originate from the parts of the depolarized region with the highest curvature, where the density of the depolarizing electrotonic current is lowest. 


\section{B. Numerical simulations of ectopic beat generation at the boundary of QSD regions}

The experimental results described above were reproduced in numerical simulations of the detailed MajumderKorhonen model of the NRVM monolayer [Fig. 2(a)]. Similar results were obtained for the detailed human TNNP ventricular model [Fig. 2(b)]. Thus, both in modeling studies and in in vitro experiments, the ectopic activity always originates at the corners of the QSD region. This

(a)
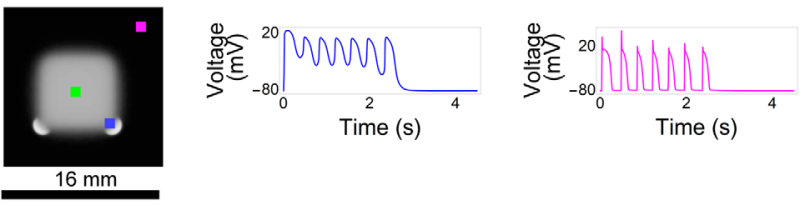

(b)
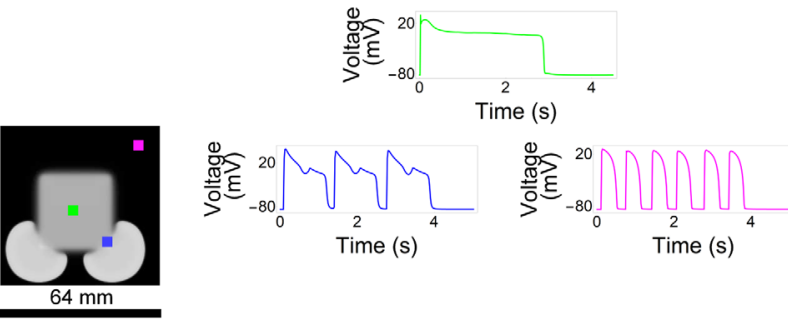

(c)
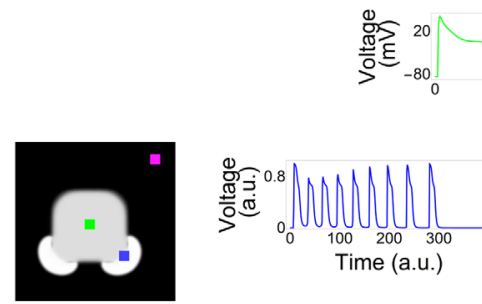

Time (a.u.)

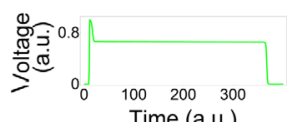

(d)

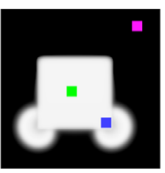

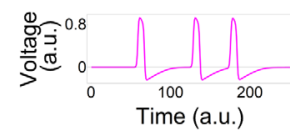

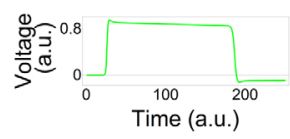

FIG. 2. Numerical modeling of ectopic wave generation from corners of a square quasistable depolarized region in four different cardiac tissue models. For each model, a snapshot of ectopic activity and voltage signals at three different locations, indicated by the green, blue, and magenta dots, are presented. (a) Majumder-Korhonen model of neonatal rat ventricular myocytes. (b) ten Tusscher-Noble-Noble-Panfilov model of adult human ventricular myocytes. Both detailed physiological models contained an increased conductance and slow inactivation variable of the late $\mathrm{Na}^{+}$current in the QSD tissue. (c) Aliev-Panfilov model. (d) FitzHugh-Nagumo model. Both of these simplified models contained a slow repolarizing variable, in addition to the bistable kinetics of the QSD tissue. In all snapshots of ectopic activity, the symmetry is broken by the first wave passing through the medium. holds true for a wide range of APDs in the QSD region; e.g., the phenomenon can be observed in silico even for QSD zones with moderately prolonged (1-1.2 s) APDs, resulting in the generation of just one ectopic beat from the corners (Fig. S5 of the Supplemental Material [18]). As also in vitro moderately prolonged APDs give rise to ectopic activity from the corners of a square QSD region (Fig. S6 of the Supplemental Material [18]), this phenomenon appears to be very robust. Although ionic models accurately reproduce the biophysical mechanism of AP generation, in a mathematical sense, they are quite complex. Thus, in order to isolate the fundamental mechanisms driving the observed phenomenon, it is always beneficial to reproduce it using a minimalistic generic description. We were able to reproduce this effect using simplified low-dimensional models, i.e., modified versions of the Aliev-Panfilov and FHN models [Figs. 2(c) and 2(d), respectively]. A detailed motivation of the simplified models choice is presented in Appendix B. As a further simplification, we were able to reproduce the same effect when the QSD region was represented as a bistable system and the normal cells as a monostable system [Fig. 3(a)], which was achieved by changing the parameter $\gamma$. This produced similar results as obtained in vitro. [Compare Figs. 1(a)-1(d) with Figs. 3(b)-3(e), respectively.] The ectopic activity originated from the corners of the bistable (i.e., QSD) region, as in the in vitro experiments. Similar data were obtained for the bistable/monostable version of the Aliev-Panfilov cardiac tissue model [22] (Fig. S2 of the Supplemental Material [18]). These results suggest that ectopic activity is more likely to occur in high-curvature border zones of the bistable (i.e., QSD) region than in border areas with low curvature. Consistently, a small discshaped bistable region yielded a propagating response in the surrounding tissue [Fig. 3(f)], but a larger QSD disc did not [Fig. 3(g)]. The fact that the effect persists even in a simple computer model implies that it is related to fundamental properties of excitation. Looking closer to the initiation mechanism of the ectopic waves, we found that the interface at the boundary of the bistable region exhibits oscillatory activity [Figs. 3(c), 3(f), and 3(g)]. In the simulation result Fig. 3(c), two oscillations of small amplitude are followed by one with a larger amplitude. The larger oscillation then forms an impulse propagating from the corner of the QSD region into the surrounding normal tissue. These numerical data strongly resemble the in vitro results of Fig. 1(b). Because of the presence of noise in the optical mapping experiments, the number of smaller amplitude oscillations needed to produce a propagating impulse in a real cardiac monolayer could not, however, be reliably determined. The importance of these oscillations was therefore scrutinized in simulations, in which the potential in the bistable region was clamped to its higher stable value $u=u_{3}$. In this case, ectopic activity from the corners disappeared. Instead, the system generated a single 
(a)

(b)
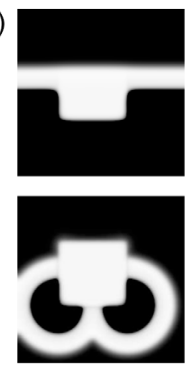

(c)

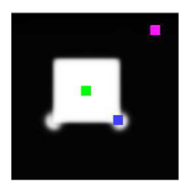

(d)

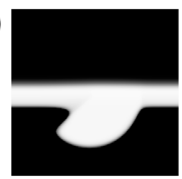

(e)

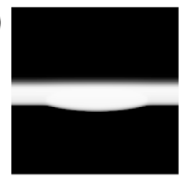

(f)

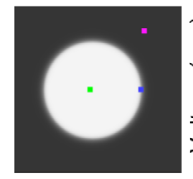

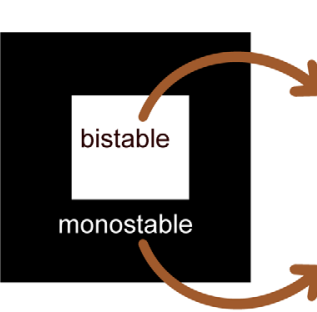

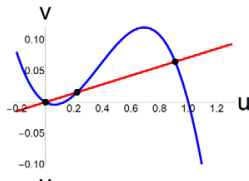

$-0.10$
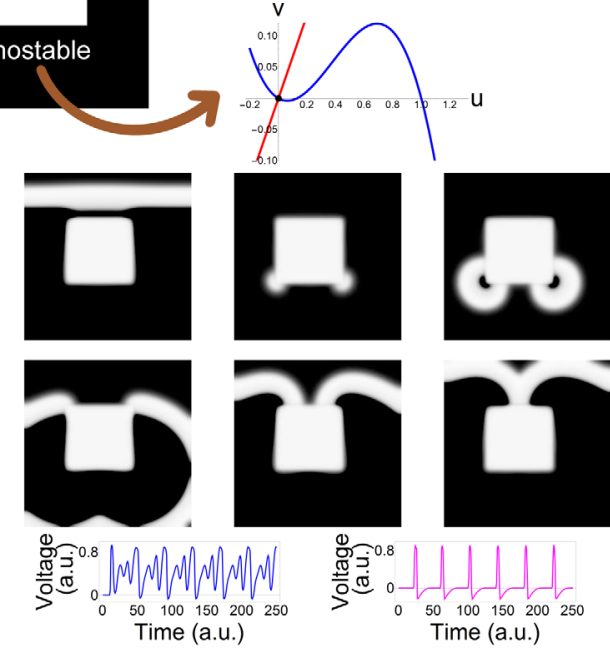

$50 \quad 100150200250$
Time (a.u.)

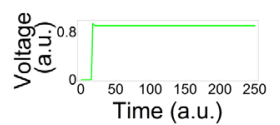
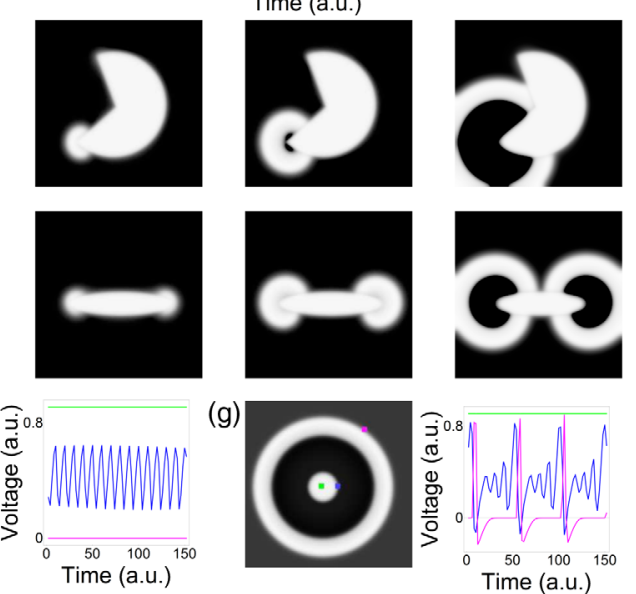

(g)
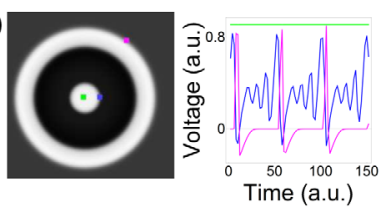

FIG. 3. Numerical modeling of ectopic waves in the FitzHughNagumo model. (a) Modeling scheme. White zones with ultralong action potentials (i.e., quasistable depolarization) are represented by a bistable system $(\gamma=14)$, while the surrounding black zone (normal tissue) is modeled as a monostable system $(\gamma=1.5)$. (b) Snapshots of ectopic beat generation from the corner of a quasistable depolarized square after passing of an initial wave [compare to Fig. 1(a)]. (c) Distribution of oscillation amplitude for oscillatory activity in (b); [compare to Fig. 1(b)]. (d),(e) Preferential generation of ectopic activity from the highest curvature areas of Pacman-shaped (d) and elliptic (e) regions [compare to Figs. 1(c) and 1(d), respectively]. The symmetry is broken by the first wave passing through the medium in (b) and (d). The wave was coming from the bottom of the domain. (f), (g) Ectopic wave potentiation by the curvature of the border zone in radially symmetric cases. Time traces, corresponding to snapshots, are indicated by green, blue, and magenta lines. (f) Subthreshold nonpropagating oscillatory response from a disc of large size (low curvature). (g) Propagating periodic response from a disc of small size (high curvature).

pulse propagating from the entire border of the heterogeneity, which was followed by the establishment of a steady-state spatial distribution of voltage (Fig. S3 of the Supplemental Material [18]).

To further study the boundary oscillations that are responsible for ectopic beat generation, we performed simulations for different nullcline slopes $\gamma_{b i}$ in the bistable region (Fig. 4), keeping the same 2D setup as in Fig. 3(a). Numerical calculations in the 2D domain were compared to 1D simulations modeling the presence of a planar QSD region. Numerically computed stationary solutions were used as initial conditions. The following changes were observed. First, for $\gamma_{b i}=20$, i.e., deep in the bistable regime, no oscillations occurred at the interface [Fig. 4(a)]. For $\gamma_{b i}=14$ [Fig. 4(b)], oscillations emerged in 1D at the interface that did not produce any waves. However, in 2D, a periodic propagating response from the corners of the square was observed. Finally, for $\gamma_{b i}=11$ [Fig. 4(c)], low amplitude oscillations occurred at the interface

(a)

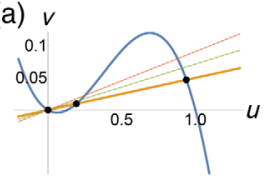

(b) $v$
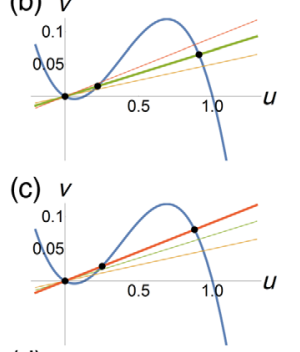

(d)

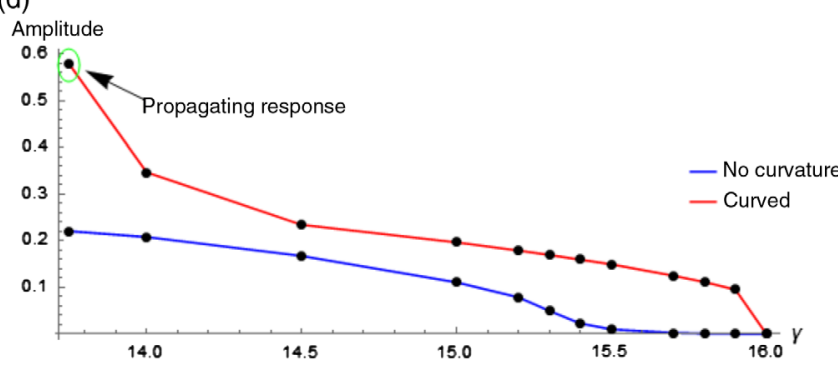

FIG. 4. Influence of the nullcline slope $1 / \gamma$ of the bistable zone on ectopic wave generation. (a)-(c) Induction of ectopic activity for $\gamma=20$ (a), 14 (b), or 11 (c). Left panels: Nullclines. Middle panels: Space-time plots of 1D simulation. Right panels: Snapshot from 2D simulation. (a) For $\gamma=20$, only the stationary solution is found in 1D and 2D. (b) For $\gamma=14$, subthreshold oscillatory activity is seen in 1D and periodic waves emanating from the corners in 2D. (c) For $\gamma=11$, a propagating response is observed both in 1D and 2D. Like in (b), the ectopic origins are located in the corners of the bistable regions. (d) Bifurcation diagram for a curved $(R=36.0$, red line) and flat (blue line) interface between the monostable (i.e., excitable) and bistable (i.e., quasistable depolarized) regions. 
between the mono- and bistable regions and periodically produced ectopic activity, both in $1 \mathrm{D}$ and in $2 \mathrm{D}$, which in the latter case arose at the corners.

By plotting the amplitude of oscillation versus $\gamma_{b i}$, the bifurcation curve for a 1D domain [Fig. 4(d), blue line] was generated. This curve showed that, at $\gamma_{b i} \leq 15.5$, oscillations with increasing amplitude arose. A similar bifurcation curve was generated for a circular depolarized region with radius $R=36.0$ (red line). In this case, the bifurcation already occurs at $\gamma_{b i}=15.9$. Thus, we again find that curvature makes the onset of instability easier, in line with the observed ectopic wave emission from corners. However, still it is not clear why curvature shifts the bifurcation point.

\section{MECHANISM OF CURVATURE-DEPENDENT INSTABILITY}

Two different approaches were chosen to explain the curvature effect: (i) the curvature-velocity relationship and (ii) the Schrödinger equation analogy by Keener and Rinzel [25].

\section{A. Qualitative explanation by backward motion of wavefront}

First, in a very general sense, the effect of positive curvature on wave propagation is a reduction of propagation velocity, which can be explained by the fact that the density of local currents for a radially expanding (i.e., convex) wavefront is smaller than that for a planar wavefront. Oppositely, negative curvature, as present in a collapsing circular (i.e., concave) wavefront, results in a more favorable source-sink relationship and, therefore, faster wave propagation than no curvature. Let us now consider a stationary distribution of voltage for the planar and curved domain and find out how the curvature of the boundary can contribute to the onset of instability. The instability can occur either by a slight shift of the stationary solution in the forward direction (i.e., towards the normal tissue) or by a similar shift backwards. The forward motion, which is similar to the expansion of a wavefront, should generally be inhibited by curvature, while backward motion should be strengthened by it. The importance of either effect can be assessed numerically, since, in the radially symmetric case, one can write the 2D Laplacian from Eq. (1) as $\Delta u=\left(\partial^{2} u / \partial r^{2}\right)+(1 / r)(\partial u / \partial r)$, where $r$ is the radial coordinate and the term $I_{\text {curv }}=(1 / r)(\partial u / \partial r)$ accounts for the wavefront curvature. We performed simulations in which $I_{\text {curv }}$ was present only in the normal or bistable region. We found that, when $I_{\text {curv }}$ was present inside the QSD region only, it enhanced the instability. For example, in the case of a circular depolarized region with a radius $R=36.0$, the ectopic waves started to appear for $\gamma_{b i}=15.3$, compared to $\gamma_{b i}=13.9$ in the fully radially symmetric system. However, in the opposite case, when
$I_{\text {curv }}$ was present outside the bistable area, the formation of ectopic activity was observed for $\gamma_{b i}<12.5$ only. Thus, under these circumstances, the curvature of the wavefront hindered the development of ectopic activity. Overall, we can conclude that informally the observed deviation from the conventional source-sink mismatch concept can be explained by the fact that curvature potentiates the initial backward motion of the wave to the inside of the QSD region, which breaks the stability of the system and eventually results in the onset of ectopic activity.

\section{B. Semianalytical study of the instability using a Schrödinger equation analogy}

To further analyze the oscillatory instability leading to ectopic activity, we extended the theoretical approach by Keener and Rinzel in Ref. [25]. However, in order to apply it, a simplification is introduced. The oscillatory instability occurs in the coupled mono- and bistable system and, thus, is present in both QSD and normal regions. In other words, the presence of the monostable region triggers activity in the border zone of the bistable region, leading to ectopic beat generation. However, in order to perform an analytical analysis, we need to restrict ourselves to one region only and replace the other region by a boundary condition. We keep the bistable QSD region and replace the normal (monostable) region by the Dirichlet boundary condition $u=0$. The rationale for this is that such a boundary condition creates a current load on the QSD region similar to that of the monostable region. Indeed, if we consider a square 2D domain in a bistable regime and Dirichlet boundary condition $u=0$ at the square's boundary, the important features of Fig. 4 will be reproduced.

In particular, for large values of $\gamma$, the solution is stable. Decreasing $\gamma$ leads to oscillations at the boundary [Fig. 5(a)], which occur at the corners and are similar to the oscillations seen in Figs. 3(c) and 4(b). Thus, once again, we see that curvature of the boundary potentiates the instability. To account for the effects of the boundary curvature, we will perform analytical calculations for the onset of instability on an interval $[l, L](0<l \ll L)$ and on an annulus with radii $[l, L]$ and compare the results. We choose an annulus instead of a disc because it simplifies the analysis and, for small $l$, the inner boundary has no effect on the onset of instability, which occurs at the outer boundary of the domain. Although our analysis can be extended to a disc, this will require additional estimations at $r=0$, which would complicate presentation of the results.

Formally, we consider Eq. (1) and impose Dirichlet boundary conditions $u(L)=0$ and $u(l)=u_{3}$ for the $1 \mathrm{D}$ case and for an annulus. In both $1 \mathrm{D}$ and $2 \mathrm{D}$, such boundary conditions allow a spatially nonuniform distribution of voltage (i.e., a smooth transition from $u=0$ to $u=u_{3}$ ), which is normally stationary in time and mimics the boundary between the QSD region and normal tissue from Fig. 3. 


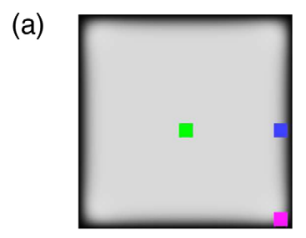

(b)

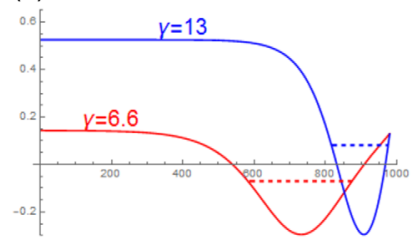

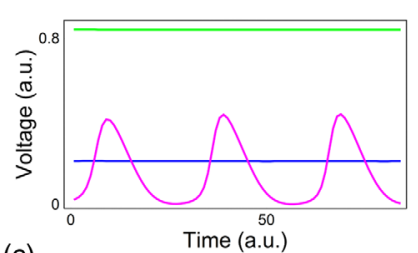

(c)

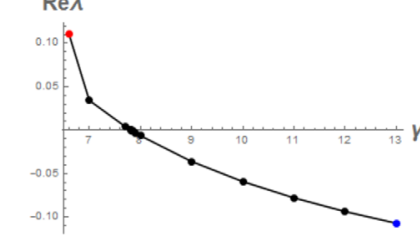

FIG. 5. (a) Localization of oscillations in corners of a bistable domain with zero Dirichlet boundary condition and $\gamma=8.9$. (b) Schrödinger potential wells with ground energy (dashed lines) values for $\gamma=6.6$ (red line) and $\gamma=13$ (blue line). (c) Stability parameter $\operatorname{Re} \lambda$ for different values of $\gamma$, from the ground energy calculation of the Schrödinger eigenvalue problem. The points corresponding to $\gamma=6.6$ and $\gamma=13$ are indicated by red and blue dots, respectively, for matching with the potential well profiles in (b).

In this case, when $\gamma$ is decreased, in the same way as in Fig. 4, we also observed the transition from stationary to oscillatory behavior, which is moreover facilitated by curvature.

The stability of the stationary solution in such a setup can be studied analytically. First, note that the stationary solutions $(u, v)=[\phi(x), \eta(x)]$ to Eq. (1) have $\eta=$ $(\phi / \gamma)$, such that, in the absence of curvature (i.e., on a line), $\phi(x)$ can be found by direct integration of the following equation, which can be viewed as Newton's equation in a potential field:

$\frac{1}{D}\left(-f(\phi)-\frac{\phi}{\gamma}\right)+\frac{\partial^{2} \phi}{\partial x^{2}}=0, \quad \phi(l)=u_{3}, \quad \phi(L)=0$,

where $u_{3}$ is the largest root of $f(u)$. Stability can be analyzed by linearizing around a stationary solution. Considering the perturbation $u=\phi+\phi_{1}, \quad v=\eta+\eta_{1}$, with $\phi_{1}=e^{\lambda t} \psi(x), \quad \eta_{1}=e^{\lambda t} z(x), \quad$ yields the stationary Schrödinger equation [25]

$$
-\frac{\partial^{2} \psi}{\partial x^{2}}+V(x) \psi=E \psi
$$

with potential $V(x)=f^{\prime}[\phi(x)] / D$ and energy $E=-[\lambda+$ $(\varepsilon / \lambda+\varepsilon \gamma)] / D$, subjected to the boundary conditions $\psi(l)=0, \psi(L)=0$. The stability of the stationary solution is lost when $\lambda$, which can be found from the energy $E=-[\lambda+(\varepsilon / \lambda+\varepsilon \gamma)] / D$, has a positive real part [25]. From the relation between $E$ and $\lambda$, one can see that decreasing $E$ leads to destabilization of the solution, a property that will be used below. Note that, since $\phi(x)$ is monotonously decreasing and $f^{\prime}$ is a quadratic function, $V(x)$ has the shape of a potential well; see Fig. 5(b).

Our numerical simulations have shown that the instability occurs when $\gamma$ is decreased below a critical value. Since a change of $\gamma$ alters the stationary solution $\phi(x)$ of Eq. (1), it affects the shape of the potential well $V(x)$, but not its depth. Representative cases are shown in Fig. 5(b) together with the numerically computed ground energy level for this potential. Lowering $\gamma$ widens the well, which decreases the energy of the ground state and, thus, facilitates the onset of instability. Figure 5(c) shows the real part of $\lambda$ as a function of $\gamma$ calculated from the ground state energy in Schrödinger's equation, predicting a critical value of $\gamma=7.813$ and a nonzero imaginary part of $\lambda$ at this value, indicating a Hopf bifurcation. We have also determined the critical value of $\gamma$ by direct numerical calculations and found it to be $\gamma=7.810$, thus very close to the theoretically predicted value. Now let us consider the effect of curvature of the domain boundary at $x=L$ (representing the edge of the depolarized region) on the onset of the instability. In this case, stationary solutions $\left\{\phi_{r}(r), \eta_{r}(r)\right\}$ can be found from

$$
\frac{1}{D}\left[-f\left(\phi_{r}\right)-\frac{\phi_{r}}{\gamma}\right]+\frac{\partial^{2} \phi_{r}}{\partial r^{2}}+\frac{1}{r} \frac{\partial \phi_{r}}{\partial r}=0 .
$$

The curvature term $(1 / r)\left(\partial \phi_{r} / \partial r\right)$ is equivalent to a friction force when Eq. (4) is interpreted as a particle in a Newtonian potential. Friction generally slows down the motion, and, in Appendix A, it is shown in detail that, as a result, $\phi_{r}(x) \leq \phi(x)$, making the potential well broader.

When performing stability analysis on this new steadystate profile $\phi_{r}$ for the radially symmetric case, we can substitute $\psi=r^{-1 / 2} \chi$, yielding

$$
-\frac{\partial^{2} \chi}{\partial r^{2}}+V_{r}(r) \chi=E \chi, \quad \chi(l)=0, \quad \chi(L)=0,
$$

with a modified potential $V_{r}(r)=-f^{\prime}\left[\phi_{r}(r)\right] / D-1 /$ $\left(4 r^{2}\right)$. The additional centrifugal term $-1 /\left(4 r^{2}\right)$ always lowers the potential well. As a result, we have shown that the spectrum of the radially symmetric problem $E_{r}$ will always be lower in comparison to the case without curvature. Therefore, in our model, positive curvature of the domain boundary will always potentiate the onset of an oscillatory (Hopf) instability.

Also, when the QSD region has a more complex shape, the Schrödinger equivalence still applies. In that case, the stationary solution will produce a potential well in two or three spatial dimensions that is localized near the boundary of the QSD region. This well will be broader in the regions where the profile of the stationary solution is less steep. As, in highly curved portions of the interface (e.g., at the corners), diffusion (i.e., electrotonic) effects are more pronounced, a local broadening of the well can be expected 
there. The Schrödinger eigenfunctions will, thus, be localized in the broader parts of the well, and these are precisely the boundary oscillations that cause the onset of instabilities. We can now understand the emergence of ectopic beats at sharp corners from a physical principle: In the corners, the transition in voltage between QSD and normal tissue will be less steep, offering more room for boundary oscillations to develop.

\section{DISCUSSION}

In this study, optogenetics has been applied to investigate the onset of ectopic activity in cardiac tissue. In our setup, the ectopic activity originated from the boundary of a QSD (ultralong AP) region. Contrary to the classical principle of source-sink mismatch, the ectopic activity preferentially arose at the boundaries between the oxidatively damaged region and the normal tissue with the highest curvature. Using in silico models and an analytical approach, we demonstrate that the mechanism of this effect is closely related to the occurrence of oscillatory (i.e., Hopf) instability at these sites.

We demonstrated such a mechanism in detailed physiological and simplified generic models. Since we reproduced the results in different models and uncovered the general underlying biophysical mechanism, we expect that our results might be applicable to different clinical situations, in which the APD is abnormally lengthened beyond $2 \mathrm{~s}$. Such situations of APD prolongation might be a result of oxidative stress [26], drug treatment, poisoning, or genetic mutations [26-29]. Compounds with strong APD-prolonging ability include $\mathrm{Ca}^{2+}$ channel agonists like Bay K8644; class Ia antiarrhythmics (e.g., quinidine); unintended hERG channel blockers like the antibiotic erythromycin; certain class III antiarrhythmics (e.g., E4031); and a large variety of neurotoxins (e.g., anemone toxin). APD-prolonging mutations have been found, e.g., in the genes encoding calmodulin [30], calmodulin-dependent kinase II [31], and fast $\mathrm{Na}^{+}$ and L-type $\mathrm{Ca}^{2+}$ channels [32,33]. APDs are also drastically increased during bradycardia induced by disease or rest and sleep $[28,29]$. The fact that APD prolongation might occur heterogeneously due to intrinsic transmural differences in repolarization kinetics, e.g., caused by increased late $\mathrm{Na}^{+}$ and decreased slowly delayed rectifier currents in the midmyocardial wall [28], further increases the proarrhythmic risk. However, as we showed experimentally (see Fig. S6 of the Supplemental Material [18]) and in numerical simulations (see Fig. S5 of the Supplemental Material [18]), extreme prolongation of $\mathrm{AP}$ is not required for ectopic wave initiation from the corners of a QSD region. It was also found for shorter APDs of $1.2 \mathrm{~s}$ in vitro, of $1 \mathrm{~s}$ in the MajumderKorhonen model of NRVMs, and of $1.5 \mathrm{~s}$ in the TNNP model of adult human ventricular cardiomyocytes. These APDs are well within the range of those described in multiple channelopathies [32,33]. APDs of $1-2 \mathrm{~s}$ can be manifested in a transient fashion during pause-induced
$[32,34]$ or rhythm-acceleration-induced $[35,36]$ APD prolongation. Both of these disturbances lead to torsade de pointes arrhythmias, which are widely observed clinically for different types of long QT syndrome [37]. To sum up, our results might apply to various clinical situations given the wide range of prolonged APDs for which the emission of ectopic waves from high curvature areas was manifested.

Besides by ultralong APs, ectopy from boundary areas of high curvature can also be caused by regional multifold increases in $\mathrm{Ca}^{2+}$ conductance. Such a local increase in $\mathrm{Ca}^{2+}$ conductance can result from long-chain fatty acid accumulation due to regional ischemia [38]. We illustrated this case in Fig. S7 of the Supplemental Material [18].

Another possible example is the ectopic activity arising from the ostia of pulmonary veins, which is one of the major triggers of atrial fibrillation. It was reported that patients with a common ostium of the left pulmonary veins have a higher propensity for developing atrial fibrillation [39]. This common ostium has an elliptic shape, thus containing border areas of high curvature, which may be a reason for the higher chance of developing arrhythmias.

Apart from providing a possible explanation for the emergence of particular forms of cardiac ectopy, our results may also help to refine surgical ablation procedures. Our finding that boundary curvature can play an important role in the generation of arrhythmic waves suggests that smoothening the borders between healthy and diseased myocardium may be beneficial, and that ablation of only the sharp convex corners of damaged cardiac tissue might be sufficient to prevent ectopic activity.

Usually, ectopic or focal activity is believed to be a result of abnormal automaticity caused by early or delayed afterdepolarizations [40] or so-called injury currents from damaged tissue [3]. For injury currents, it has been reported that abnormal automaticity may originate from the coupling of an excitable cell with another cell with a higher (i.e., less negative) resting membrane potential [41-43]. Despite the fact that the resting states of both cells are stable, an oscillatory (i.e., Hopf) bifurcation can emerge in such a coupled system. A similar situation exists in Figs. 1(c) and 3(c). However, in our case, the Hopf bifurcation emerges from the coupling of multiple mono- and bistable cells in a diffusive manner.

Keener and Rinzel [25] were the first to identify this Hopf instability in a FHN system for the case of a single fiber with Dirichlet or Neumann boundary conditions at one of the ends. Here, we have extended their approach to curved domains.

Similar behavior has been observed for the interface between oscillatory and excitable regions in the BelousovZhabotinsky reaction $[44,45]$. However, no local bifurcation analysis was possible for simulation of this chemical activity, since the limit cycles already had a high amplitude.

In physical systems, curvature-induced effects have been observed in nonlinear optics of solitons [46,47], shock 
waves in Bose-Einstein condensate [48], the appearance of additional curvature-induced magnetic force in a magnetic shell [49], the onset of superconductivity in curved domains $[50,51]$, the appearance of prohibited states in condensed matter physics and material science [52], and curvature-induced bound states for quantum wires [53]. In all these cases, positive curvature induced unusual behavior, which cannot be observed under normal conditions in a flat geometry. The last example is most striking and stems from the fundamental result of topological trapping of a quantum particle [54]. In Ref. [54], it was shown that a quantum mechanical particle is preferentially trapped in a region of high curvature of a spatially extended Schrödinger well. The same principle applied here: The strong curvature near corners of the QSD region lowers the energy of the Schrödinger well precisely there. Overall, it is not surprising to find a quantum mechanical analogy in a reaction-diffusion context due to the long-standing mathematical similarity of the diffusion and Schrödinger equations [55]. One can just go to imaginary time and get the similar retrograde diffusion equations. Similarity between the diffusion formalism and Schrödinger equation can also be found on more general physical grounds due to the analytical continuation connection between the Wiener integral for the Brownian motion process and the Feynman path integral [56]. Therefore, the quantum mechanical analogy of localization might be applicable to more complex cases of multiple diffusing species [44] in comparison to the simple diffusion of voltage in our case. Because of the simplicity and generality of our computer model, we would expect similar effects to be found in other areas of biology, chemistry, and physics.

Albeit we reproduced the phenomenon in complex detailed models, in our research we have intentionally focused on a simplified computer model for cardiac tissue in order to relate the effect to the most fundamental properties of cardiac excitation and to be able to perform an analytical study of the dynamics. It would, however, also be of interest to investigate the role of different ionic currents in the observed effects in a more detailed mathematical model of cardiac muscle cells.

In our paper, we were able to reproduce the experimentally observed effect of ectopy from the corners in a highly relevant mathematical model for our experimental system, namely, the Majumder-Korhonen model of NRVMs and in a detailed and more clinically relevant model of adult human ventricular cardiomyocytes. Although, in all our in silico models, ectopy from boundary areas with high curvature occurs for a wide range of parameters (for example, for the QSD zone, as shown in Fig. 4, $11 \leq \gamma_{b i} \leq 14$ results in corner-confined ectopy), we did not study in detail all possible regimes in such a system, e.g., ectopy from corners, faces, or middles of the region, and the locations of such regimes in the parametric space. It would, hence, be interesting to investigate all possible manifestations of ectopy in a wider class of mathematical models, including detailed models for human cardiac tissue (see, e.g., O'HaraRudy [57] and Grandi [58]) and modern low-dimensional cardiac models (see, e.g., Mitchell-Schaeffer [59] and Corrado [60]) in future studies.

The extension of our work to 3D would be of importance as well, since injured cardiac ventricles typically exhibit a complex 3D structure. The effects of curvature on ectopic activity are expected to be even more pronounced in $3 \mathrm{D}$ systems than in our 2D preparations.

In conclusion, we have demonstrated, using complementary in vitro and in silico models, that the border zones between damaged and healthy myocardium with the highest positive curvature are the most likely areas for the onset of ectopic activity. As the electrotonic load for formation of excitation is maximal in such areas, this effect is paradoxical. Our finding, thus, adds a new potential mechanism for cardiac ectopy and represents an additional controllable degree of freedom to prevent arrhythmogenesis.

\section{ACKNOWLEDGMENTS}

We thank Annemarie Kip, Cindy Bart, Marc Engels, and Jia Liu for excellent technical support and help in producing lentiviral vector particles and Iolanda Feola for guidance in preparing NRVM monolayers. We also thank Rupamanjari Majumder and Nina Kudryashova for fruitful discussions. This work was supported by the Netherlands Organization for Scientific Research (NWO Vidi Grant No. 917143 to D. A.P.). A. S. T., D. A.P., and A. V.P. initiated and designed the research. A.S.T. performed experiments and computations. A.S.T., H.D., and A. V.P. analyzed data and conceived theoretical explanations. A. A. F. V. designed genetic engineering tools. A. A. F. V. and D. A. P. provided reagents; D. A. P. provided funding sources; and A. S. T., H. D., A. V.P, A. A. F. V., and D. A.P. wrote the paper.

\section{APPENDIX A: ANALYTICAL PROOF THAT CURVATURE PROMOTES INSTABILITY}

Our proof will be based on the Schrödinger analogy first given by Rinzel and Keener. They stated that the relation $E(\lambda)$ can be inverted by solving the quadratic equation

$$
\lambda^{2}+(\epsilon \gamma+D E) \lambda+\epsilon(1+\gamma D E)=0 .
$$

From studying the possible locations of roots $\lambda_{1}, \lambda_{2}$ in the complex plane, it follows that at least one root has a positive real part if and only if $\lambda_{1}+\lambda_{2}=-(\epsilon \gamma+D E)$ is positive. Therefore, the stationary solution will become unstable when $E<E_{0}=-\epsilon \gamma / D$. At this critical value, $\lambda_{1} \lambda_{2}=$ $\left(1+\gamma D E_{0}\right)=1-\gamma^{2} \epsilon>0$ in our working regime. This indicates that, at the instability threshold, $\lambda_{1}, \lambda_{2}$ are purely imaginary and, therefore, the system undergoes a Hopf bifurcation. 
We now proceed to show that positive curvature of the boundary promotes instability of the system by proving that positive curvature of the boundary always lowers $E$.

Adding curvature to the system changes both the stationary profile and the associated linearized equation. We show this in two steps. First, we write the systems [1] and [3] of the main text as a particle with unit mass in a Newtonian potential: letting $u$ correspond with 1D particle position $X$ and $x$ with time $t \in[l, L]$ yields

$$
\frac{d^{2} X}{d t^{2}}+b(t) \frac{d X}{d t}=-\frac{d U(X)}{d X},
$$

with potential $U(X)=\int^{X} f(\phi) d \phi$. For the case without curvature, $b(t)=0$, corresponding to a frictionless Newtonian system; the case with curvature is equivalent to the particle being subjected to a frictional force $b(t)=1 / t$. In both cases, the boundary conditions are $X(l)=u_{3}$ and $X(L)=u_{1}$. A representative solution for both cases is given in Fig. S4(a) of the Supplemental Material [18]. Note that, since both particles need to travel the same distance $u_{3}-u_{1}$ in the same time interval $L-l$, the frictionless case starts with a smaller initial velocity and, therefore, always lags behind the case with friction, only catching up at time $t=L$. Since $u_{3}>u_{1}$ and the Newtonian paths were called $\phi(x)$ and $\phi_{r}(x)$ in the main text, we thus have

$$
\phi(x)>\phi_{r}(x), \quad l<x<L .
$$

With this result, we can compare the eigenvalues $E$ of the linear system,

$$
-\frac{\partial^{2} \psi}{\partial x^{2}}+V(x) \psi=E \psi,
$$

for potentials $V(x)=-f^{\prime}(\phi) / D$ or $V_{r}(x)=-f^{\prime}\left(\phi_{r}\right) / D$, yielding ground state eigenvalues $E$ and $E_{1}$, respectively. Note that the term $(1 / x)(d \psi / d x)$ is not included here yet; it will be performed below. A well-known property of the Schrödinger problem is that a wider and deeper well lowers the energy. For the two cases considered, the well depth is equal to $-f^{\prime}\left(u_{m}\right) / D$, with $u_{m}$ representing the unique inclination point of $f(u)$. Thus, the difference in $E$ will only depend on the width of the potential well. To define the well width $W_{r}$ or $W$ for the cases with and without curvature, we consider the typical wells for our system as shown in Figs. 5(b) and in S4(b) of the Supplemental Material [18]. Note that $f^{\prime}(\phi)$ is a quadratic function centered at $\phi=u_{m}<\left(u_{1}+u_{3}\right) / 2$; see Fig. S4(c) of the Supplemental Material [18]. Hence, $V(l)>V(L)$; i.e., the potential is always higher on the left-hand side, where the boundary condition is $u(l)=u_{3}$. Therefore, it makes sense to define the width of the potential as the region where $V(x)<V(L)$; see Fig. S4(a) of the Supplemental
Material [18]. Since $f^{\prime}(u)$ is a quadratic function and $u_{1}=0$, it follows that $f^{\prime}\left(2 u_{m}\right)=V(L)$, such that $W=$ $L-\phi^{-1}\left(2 u_{m}\right), W_{r}=L-\phi_{r}^{-1}\left(2 u_{m}\right)$. Hence, it follows from Eq. (A3) that

$$
W_{r}>W \Rightarrow E_{1}<E \text {. }
$$

We still have to consider the effect of adding the curvature term to Eq. (A4). However, in the main text, it is shown that a substitution $\psi=r^{-1 / 2} \chi$ produces again the Schrödinger problem with a potential shifted down by $1 /\left(4 r^{2}\right)$ and eigenvalue $E_{r}$. Therefore,

$$
E_{r}<E_{1}<E .
$$

Hence, we have established that positive curvature at the boundary of a bistable domain promotes instability. As we have only used qualitative properties of the solution, e.g., monotonicity of the profile and the asymmetric sigmoidal shape of $f(u)$, our analysis is not restricted to the FHN model. Our analysis can be extended for a disc. This would, however, require additional estimations at $r=0$ that would unnecessarily complicate the presentation of the results.

\section{APPENDIX B: SUPPLEMENTARY MATERIALS AND METHODS}

\section{Cell isolation and culture}

NRVMs were isolated and cultured as reported previously [16]. In brief, hearts were excised from neonatal rats under anaesthesia, and venticular tissue was delicately chopped and dissociated with $450 \mathrm{U} / \mathrm{ml}$ collagenase type I (Worthington, Lakewood, New Jersey) and 18.75 Kunitz/ml DNase I (Sigma-Aldrich, St. Louis, Missouri). Cells were seeded on round, fibronectin-coated (Sigma-Aldrich) 15-mm-diameter glass coverslips in 24well culture plates (Corning Life Sciences, Corning, New York). Cells were seeded at a density of $8 \times 10^{5}$ cells/well and incubated for $2 \mathrm{~h}$ with mitomycin-C $(10 \mu \mathrm{g} / \mathrm{ml}$, Sigma-Aldrich) to inhibit proliferation of nonmyocytes.

\section{Molecular cloning}

The self-inactivating lentiviral shuttle plasmid pLV.hCMV-IE.miniSOG-PM.hHBVPRE, which codes for a plasma-membrane-associated version of miniSOG, was engineered by a two-step operation. In step 1, the miniSOGcoding sequence was extended with the plasma-membrane targeting motif of human K-Ras4B by substituting the BglII $\times$ EcoRI fragment of plasmid miniSOG-C1 [15] with a linker molecule composed of oligonucleotides 5' GATCCAAGATGAGCAAAGACGGCAAAAAGAAGAAAAAGAAGTCCAAGACAAAGTGCGTGATCATGTAAAG 3' and 5' AATTCTTTACATGATCACGCACTTTGTCTTGGACTCTTTTTCTTCTTTTTGCCGTCTTTGCTCATCTTG 3' (Sigma-Aldrich). The resulting plasmid was designated 
pminiSOG-PM. In step 2, the 414-bp Eco47III $\times$ EcoRI fragment of pminiSOG-PM was blunt-ended with Klenow polymerase and inserted in between the SmaI site and filledin BsrGI site of pLV.hCMV-IE.IRES.eGFP.hHVBPRE [16]. The resulting construct was designated pLV.hCMVIE.miniSOG-PM.hHBVPRE. The restriction enzymes and other DNA-modifying enzymes were purchased from New England Biolabs (Bioké, Leiden, the Netherlands) or Thermo Fisher Scientific (Landsmeer, the Netherlands). Large-scale plasmid isolation was performed with the JETSTAR 2.0 Plasmid Maxiprep kit (Genomed, Löhne, Germany), in accordance with the instructions supplied with the kit.

\section{Lentiviral vector particle production and transduction of confluent monolayers of NRVMs}

Construct pLV.hCMV-IE.miniSOG-PM.hHBVPRE was used for the production of miniSOG-encoding lentiviral vector particles employing a second-generation packaging system as previously detailed [17]. These particles were subsequently used to transduce 5-day-old continuous monolayer cultures of NRVMs with a vector dose that resulted in transduction of approximately $95 \%$ of the cells. Assessment of the transduction efficiency was done with an inverted phase-contrast and fluorescence microscope (Axiovert 35, Carl Zeiss, Sliedrecht, the Netherlands) by visualization of the green fluorescent signals produced by excited miniSOG molecules.

\section{Optical mapping and patterned illumination of monolayers}

After 8-10 days of culturing, ventricular monolayers were optically mapped using the voltage-sensitive dye di-4ANEPPS (Thermo Fisher Scientific) as reported previously [17]. The mapping setup was based on a $100 \times 100$ pixel CMOS Ultima-L camera (Scimedia, Costa Mesa, California). The field of view was $16 \times 16 \mathrm{~mm}$, resulting in a spatial resolution of $160 \mu \mathrm{m} /$ pixel. For targeted illumination of monolayers, the setup was optically conjugated to a digitally controlled micromirror device (DMD), the Polygon 400 (Mightex Systems, Toronto, Ontario), with a high-power blue (470-nm) LED (BLS-LCS-0470-5022-H, Mightex Systems). Before starting the actual experiments, all monolayers were mapped during $1-\mathrm{Hz}$ electrical point stimulation to check baseline conditions. Electrical stimulation was performed by applying 10-ms-long rectangular electrical pulses with an amplitude of $8 \mathrm{~V}$ to a bipolar platinum electrode with a spacing of $1.5 \mathrm{~mm}$ between the anode and cathode. Only cultures with an APD at $80 \%$ repolarization $\left(\mathrm{APD}_{80}\right)$ below $350 \mathrm{~ms}$ and a conduction velocity above $18 \mathrm{~cm} / \mathrm{s}$ were used for further experiments. Next, monolayers were illuminated with different light patterns at constant intensity $\left(0.3125 \mathrm{~mW} / \mathrm{mm}^{2}\right)$ in the sample plane for 3-6 min. The resulting electrical activity was recorded for 6-24s at exposure times of 1 or $6 \mathrm{~ms}$ per frame.

\section{Data analysis}

Data analysis was performed using specialized BV Ana software (Scimedia), ImageJ Ref. [61], and custom-written scripts in Wolfram Mathematica (Wolfram Research, Hanborough, Oxfordshire, United Kingdom). APD and conduction velocity were calculated as described previously [17]. To prepare representative frames of wave propagation, optical mapping videos were filtered with a spatial averaging filter $(3 \times 3$ stencil $)$ and a derivative filter.

\section{Numerical modeling and calculations}

\section{a. Numerical calculations with simplified models}

For 2D and 1D simulations, we used the forward Euler method with time step $\Delta t=0.002$ and a centered finitedifferencing scheme to discretize the Laplacian with space step $\Delta x=0.25$. A $1024 \times 1024$ grid was used for the $2 \mathrm{D}$ simulation and a 1024-point cable for the 1D simulation. In all simplified models, spatially uniform zero initial conditions were used for all variables. To eliminate possible spatial discretization errors, we used time step $\Delta t=$ 0.00015625 and space step $\Delta x=0.03125$, and we used 8192 grid points for the 1D bistable system and for the Schrödinger eigenvalue problem. The Schrödinger eigenvalue problem was solved by calculating a finitedimensional matrix eigenvalue problem using a centered second-order finite-differencing scheme for the Laplacian operator.

\section{b. Aliev-Panfilov model}

We used the modified Aliev-Panfilov model [22] to create bistable and monostable zones as shown in Fig. S2 of the Supplemental Material [18],

$$
\begin{aligned}
& \frac{\partial u}{\partial t}=-k(u-1)(u-a)-r u v+D \Delta u, \\
& \frac{\partial v}{\partial t}=0.9\left(c+\left(\frac{d_{1} v}{d_{2}+u}\right)\right)[-v-k u(u-b-1)],
\end{aligned}
$$

where $c=0.002, b=0.15, d_{1}=0.2, d_{2}=0.3, k=8$, and $D=2.0$. These parameters are the same for the bi- and monostable zone. The equations for the monostable zone were converted to bistable ones by using the threshold $a=$ 0.08 and a repolarizing force $r=0.61$. The values for the monostable zone were $a=0.15$ and $r=1$ as in the original Aliev-Panfilov formulation. The corresponding nullclines are shown in Fig. S2(a) of the Supplemental Material [18].

\section{c. Implementation of ultralong APs in the FHN model}

To mimic ultralong APs in a simplified model, we modified the FHN reaction-diffusion model as follows: 


$$
\begin{aligned}
& \frac{\partial u}{\partial t}=-f(u)-v-g+D \Delta u, \\
& \frac{\partial v}{\partial t}=\varepsilon[u-\gamma(\vec{r}) v], \\
& \frac{\partial g}{\partial t}=\varepsilon_{2}\left(u-\gamma_{2} g\right) .
\end{aligned}
$$

Here, $f(u)=u(u-1)(u-a), a=0.13, \varepsilon=0.004$, and $D=2.0$. The state variable $g$ was added to the standard model, having the slowest timescale $\varepsilon_{2}=0.00005 \ll$ $\varepsilon \ll 1$. Another parameter $\gamma_{2}=3.12$. For $g=0$, the differential equations for $u$ and $v$ constitute a monostable or bistable system. Normal tissue was modeled as a monostable system $\left(\gamma=\gamma_{\text {mono }}=1.5\right)$, if $g=0$. QSD tissue was modeled as a bistable system $\left(\gamma=\gamma_{b i}=14.0\right)$, if $g=0$.

\section{d. Implementation of ultralong APs in the Aliev-Panfilov model}

Similarly to the previous paragraph, we introduced a very slowly recovering variable $g$ in the Aliev-Panfilov model:

$$
\begin{aligned}
& \frac{\partial u}{\partial t}=-k(u-1)(u-a)-r u(v+g)+D \Delta u \\
& \frac{\partial v}{\partial t}=0.9\left(c+\left(\frac{d_{1} v}{d_{2}+u}\right)\right)[-v-k u(u-b-1)] \\
& \frac{\partial g}{\partial t}=\varepsilon_{2}\left(u-\gamma_{2} g\right)
\end{aligned}
$$

where $\varepsilon_{2}=0.0001, \gamma_{2}=3.12$.

\section{e. Detailed electrophysiological model of NRVMs with regionally activated late $\mathrm{Na}^{+}$current}

The monolayer model of NRVMs was adopted from [19]. The steady-state voltage dependence for the inactivation variable $h$ of fast $\mathrm{Na}^{+}$current was changed to $h_{\infty}=\left[1+e^{[(65+V) / 6.07]}\right]^{-1}$, where $V$ is the transmembrane potential. In the square QSD zone, the late $\mathrm{Na}^{+}$current was formulated as an additional low-conductance $\mathrm{Na}^{+}$current, in accordance with [62]

$$
I_{\mathrm{Na}}=G_{\mathrm{NaL}} m_{L}^{3} h_{L}\left(V-E_{\mathrm{Na}}\right),
$$

where $G_{\mathrm{NaL}}=1.2288 \mathrm{mS} / \mu \mathrm{F}$. The formulation of the activation variable $m_{L}$ coincides with the formulation of $m$ for the fast $\mathrm{Na}^{+}$current and, for $h_{L}$, it is given by

$$
\frac{d h_{L}}{d t}=\frac{h_{L}-h_{L, \infty}}{\tau_{h L}} .
$$

Here, the steady-state voltage dependence was chosen to be $h_{L, \infty}=\left[1+e^{[(101+V) / 6.1]}\right]^{-1}$. By changing time constant $\tau_{h L}$, we were able to modify the duration of ectopic activity episodes. $\tau_{h L}=15000 \mathrm{~ms}$ was chosen for Fig. 2 and $\tau_{h L}=$ $3000 \mathrm{~ms}$ for Fig. S5(a) of the Supplemental Material [18].
The forward Euler method was used to integrate the equations with a time step $\Delta t=0.005 \mathrm{~ms}$ and a centered finite-differencing scheme to discretize the Laplacian with a space step of $\Delta x=0.0625 \mathrm{~mm}$. The total computational domain size was $256 \times 256$ grid points; the centrally located square with the activated late $\mathrm{Na}^{+}$current consisted of $115 \times 115$ grid points. To create stationary initial conditions, the model was integrated for $2 \mathrm{~min}$ before a single stimulus was applied to the lower border of the domain.

\section{f. Detailed electrophysiological model of adult human ventricular myocytes with regionally activated late $\mathrm{Na}^{+}$current}

The ten Tusscher-Noble-Noble-Panfilov model was adopted from Ref. [20]. The conductances of the transient outward $\left(G_{\mathrm{to}}\right)$, rapid $\left(G_{\mathrm{Kr}}\right)$, and the slow delayed $\left(G_{\mathrm{Ks}}\right)$ rectifier $K^{+}$current were reduced to $50 \%$ of their original values. This did not significantly prolong APD.

The late $\mathrm{Na}^{+}$current was implemented in the same manner as for the rat model. We used the same formalism as in Eq. (B4), where the activation kinetics coincides with the kinetics of the fast $\mathrm{Na}^{+}$current. The inactivation variable also followed Eq. (B5). The steady-state inactivation was $h_{L, \infty}=\left[1+e^{[(101+V) / 6.1]}\right]^{-1}$, where $V$ is the transmembrane potential; the conductance of the channel $G_{\mathrm{NaL}}=$ $0.65536 \mathrm{mS} / \mu \mathrm{F}$. Time constants were $\tau_{h L}=25000 \mathrm{~ms}$ for the prolonged episode in Fig. 2 of the main manuscript and $\tau_{h L}=5000 \mathrm{~ms}$ for the short episode in Fig. S5(b) of the Supplemental Material [18]. The forward Euler method was used to integrate the equations with a time step $\Delta t=0.005 \mathrm{~ms}$ and a centered finite-differencing scheme to discretize the Laplacian with a space step $\Delta x=0.0625 \mathrm{~mm}$. The diffusion coefficient was equal to $0.000154 \mathrm{~cm}^{2} / \mathrm{ms}$. The total computational domain size was $1024 \times 1024$ grid points; the centrally located square with the activated late $\mathrm{Na}^{+}$current consisted of $460 \times 460$ grid points. The model was integrated for $2 \mathrm{~min}$ to create stationary initial conditions. Thereafter, a single pulse was delivered at the lower border of the domain.

\section{g. Detailed electrophysiological model of adult human ventricular myocytes with regionally increased $\mathrm{Ca}^{2+}$ current}

The ten Tusscher-Noble-Noble-Panfilov model was adopted from Ref. [20]. The conductance of the transient rapid delayed rectifier $\left(G_{\mathrm{Kr}}\right)$ was set to zero throughout the computational domain. The time constant of the inactivating $f$-gate of the L-type $\mathrm{Ca}^{2+}$ was reduced twofold uniformly in the domain. The conductance of L-type $\mathrm{Ca}^{2+}$ channel $G_{\mathrm{CaL}}$ was increased 12 times inside the square to mimic damaged tissue and 2 times in the surrounding tissue, causing a sixfold difference in conductance between the inner and outer regions of the domain. The diffusion coefficient was set 
to $0.000154 \mathrm{~cm}^{2} / \mathrm{ms}$. The forward Euler method was used to integrate the equations with $\Delta t=0.005 \mathrm{~ms}$. The Laplacian was implemented using a centered finite-differencing scheme with $\Delta x=0.0625 \mathrm{~mm}$. The total domain size was $512 \times 512$ grid points; the centrally located square with the increased $\mathrm{Ca}^{2+}$ current consisted of $230 \times 230$ grid points. The model was integrated for 2 min to create stationary initial conditions, after which a stimulus was delivered at the lower domain boundary. The initial wave of excitation led to a sustained depolarization of the square, followed by ectopic waves originating from the corners of the square (Fig. S7 of the Supplemental Material [18]).

[1] R. Mehra, Global Public Health Problem of Sudden Cardiac Death, J. Electrocardiol. 40, S118 (2007).

[2] S. M. Pogwizd, R. H. Hoyt, J. E. Saffitz, P. B. Corr, J. L. Cox, and M.E. Cain, Reentrant and Focal Mechanisms Underlying Ventricular Tachycardia in the Human Heart, Circulation 86, 1872 (1992).

[3] M. J. Janse, F. J. Van Capelle, H. Morsink, A. G. Kléber, F. Wilms-Schopman, R. Cardinal, C. N. d'Alnoncourt, and D. Durrer, Flow of "Injury" Current and Patterns of Excitation During Early Ventricular Arrhythmias in Acute Regional Myocardial Ischemia in Isolated Porcine and Canine Hearts. Evidence for Two Different Arrhythmogenic Mechanisms, Circ. Res. 47, 151 (1980).

[4] A. T. Winfree, The Geometry of Biological Time (Springer Science \& Business Media, New York, 2001), Vol. 12.

[5] H. Dierckx, O. Bernus, and H. Verschelde, Accurate Eikonal-Curvature Relation for Wave Fronts in Locally Anisotropic Reaction-Diffusion Systems, Phys. Rev. Lett. 107, 108101 (2011).

[6] J. W. Cahn and J. E. Hilliard, Free Energy of a Nonuniform System. III. Nucleation in a Two-Component Incompressible Fluid, J. Chem. Phys. 31, 688 (1959).

[7] P. W. Bates and P. C. Fife, The Dynamics of Nucleation for the Cahn-Hilliard Equation, SIAM J. Appl. Math. 53, 990 (1993).

[8] A. G. Kléber and Y. Rudy, Basic Mechanisms of Cardiac Impulse Propagation and Associated Arrhythmias, Physiol. Rev. 84, 431 (2004).

[9] A. Arutunyan, D. R. Webster, L. M. Swift, and N. Sarvazyan, Localized Injury in Cardiomyocyte Network: A New Experimental Model of Ischemia-Reperfusion Arrhythmias, Am. J. Physiol. 280, H1905 (2001).

[10] K. R. Pitts and C. F. Toombs, Coverslip Hypoxia: A Novel Method for Studying Cardiac Myocyte Hypoxia and Ischemia In Vitro, Am. J. Physiol. 287, H1801 (2004).

[11] E. S. Boyden, F. Zhang, E. Bamberg, G. Nagel, and K. Deisseroth, Millisecond-Timescale, Genetically Targeted Optical Control of Neural Activity, Nat. Neurosci. 8, 1263 (2005).

[12] M. Watanabe, I. Feola, R. Majumder, W. Jangsangthong, A. S. Teplenin, D. L. Ypey, M. J. Schalij, K. Zeppenfeld, A. A. de Vries, and D. A. Pijnappels, Optogenetic Manipulation of Anatomical Re-entry by Light-Guided Generation of a Reversible Local Conduction Block, Cardiovasc. Res. 113, 354 (2017).

[13] H. M. McNamara, H. Zhang, C. A. Werley, and A.E. Cohen, Optically Controlled Oscillators in an Engineered Bioelectric Tissue, Phys. Rev. X 6, 031001 (2016).

[14] A. Teplenin, W. Jangsangthong, I. Feola, M. Schalij, D. Ypey, A. De Vries, and D. Pijnappels, Microfoci of Oxidative Stress Increase Pro-arrhythmic Risk as Revealed by Patterned Illumination of Optogenetically Engineered Myocardial Cultures, European Heart Journal 37, 711 (2016).

[15] X. Shu, V. Lev-Ram, T. J. Deerinck, Y. Qi, E. B. Ramko, M. W. Davidson, Y. Jin, M. H. Ellisman, and R. Y. Tsien, A Genetically Encoded Tag for Correlated Light and Electron Microscopy of Intact Cells, Tissues, and Organisms, PLoS Biol. 9, e1001041 (2011).

[16] M. C. Engels, S. F. Askar, W. Jangsangthong, B. O. Bingen, I. Feola, J. Liu, R. Majumder, M. I. Versteegh, J. Braun, R. J. Klautz et al., Forced Fusion of Human Ventricular Scar Cells with Cardiomyocytes Suppresses Arrhythmogenicity in a Co-culture Model, Cardiovasc. Res. 107, 601 (2015).

[17] I. Feola, A. Teplenin, A. A. de Vries, and D. A. Pijnappels, in Optogenetic Engineering of Atrial Cardiomyocytes, Optogenetics. Methods in Molecular Biology Vol. 1408, edited by A. Kianianmomeni (Humana Press, New York, 2016), pp. 319-331.

[18] See Supplemental Material at http://link.aps.org/ supplemental/10.1103/PhysRevX.8.021077 for Supplementary Figures 1-7.

[19] R. Majumder, M. C. Engels, A. A. De Vries, A. V. Panfilov, and D. A. Pijnappels, Islands of Spatially Discordant APD Alternans Underlie Arrhythmogenesis by Promoting Electrotonic Dyssynchrony in Models of Fibrotic Rat Ventricular Myocardium, Sci. Rep. 6, 24334 (2016).

[20] K. H. ten Tusscher, D. Noble, P.-J. Noble, and A. V. Panfilov, A Model for Human Ventricular Tissue, Am. J. Physiol. 286, H1573 (2004).

[21] R. FitzHugh, Impulses, and Physiological States in Theoretical Models of Nerve Membrane, Biophys. J. 1, 445 (1961).

[22] R. R. Aliev and A. V. Panfilov, A Simple Two-Variable Model of Cardiac Excitation, Chaos Solitons Fractals 7, 293 (1996).

[23] V. Zykov, A. Krekhov, and E. Bodenschatz, Fast Propagation Regions Cause Self-Sustained Reentry in Excitable Media, Proc. Natl. Acad. Sci. U.S.A. 114, 1281 (2017).

[24] J. Keener and J. Sneyd, Mathematical Physiology: I: Cellular Physiology (Springer Science \& Business Media, New York, 2010).

[25] J. Rinzel and J. P. Keener, Hopf Bifurcation to Repetitive Activity in Nerve, SIAM J. Appl. Math. 43, 907 (1983).

[26] J. C. Shryock, Y. Song, S. Rajamani, C. Antzelevitch, and L. Belardinelli, The Arrhythmogenic Consequences of Increasing Late INa in the Cardiomyocyte, Cardiovasc. Res. 99, 600 (2013).

[27] L. Belardinelli, W. R. Giles, S. Rajamani, H. S. Karagueuzian, and J. C. Shryock, Cardiac Late $\mathrm{Na}^{+}$Current: Proarrhythmic Effects, Roles in Long QT Syndromes, and Pathological Relationship to CaMKII and Oxidative Stress, Heart Rhythm 12, 440 (2015).

[28] C. Antzelevitch, M Cells in the Human Heart, Circ. Res. 106, 815 (2010). 
[29] Z. Qu and D. Chung, Mechanisms and Determinants of Ultralong Action Potential Duration and Slow Rate-Dependence in Cardiac Myocytes, PLoS One 7, e43587 (2012).

[30] B. A. Alseikhan, C. D. DeMaria, H. M. Colecraft, and D. T. Yue, Engineered Calmodulins Reveal the Unexpected Eminence of $\mathrm{Ca} 2+$ Channel Inactivation in Controlling Heart Excitation, Proc. Natl. Acad. Sci. U.S.A. 99, 17185 (2002).

[31] O. M. Koval, X. Guan, Y. Wu, M.-1. Joiner, Z. Gao, B. Chen, I. M. Grumbach, E. D. Luczak, R. J. Colbran, L.-S. Song et al., CaV $1.2 \beta$-Subunit Coordinates CaMKII-Triggered Cardiomyocyte Death and Afterdepolarizations, Proc. Natl. Acad. Sci. U.S.A. 107, 4996 (2010).

[32] J. D. Moreno, P.-C. Yang, J. R. Bankston, E. Grandi, D. M. Bers, R. S. Kass, and C. E. Clancy, Ranolazine for Congenital and Acquired Late INa Linked Arrhythmias: In Silico Pharmacologic Screening, Circ. Res. 113, e50 (2013).

[33] X. Huang, T. Y. Kim, G. Koren, B.-R. Choi, and Z. Qu, Spontaneous Initiation of Premature Ventricular Complexes and Arrhythmias in Type 2 Long QT Syndrome, Am. J. Physiol. 311, H1470 (2016).

[34] P. C. Viswanathan and Y. Rudy, Pause Induced Early Afterdepolarizations in the Long QT Syndrome: A Simulation Study, Cardiovasc. Res. 42, 530 (1999).

[35] A. Burashnikov and C. Antzelevitch, Acceleration-Induced Action Potential Prolongation and Early Afterdepolarizations, J. Cardiovasc. Electrophysiol. 9, 934 (1998).

[36] D. Nuyens, M. Stengl, S. Dugarmaa, T. Rossenbacker, V. Compernolle, Y. Rudy, J. F. Smits, W. Flameng, C. E. Clancy, L. Moons et al., Abrupt Rate Accelerations or Premature Beats Cause Life-Threatening Arrhythmias in Mice with Long-QT3 Syndrome, Nat. Med. 7, 1021 (2001).

[37] M. O. Sweeney, L. L. Ruetz, P. Belk, T. J. Mullen, J. W. Johnson, and T. Sheldon, Bradycardia Pacing-Induced Short-Long-Short Sequences at the Onset of Ventricular Tachyarrhythmias: A Possible Mechanism of Proarrhythmia?, J. Am. Coll. Cardiol. 50, 614 (2007).

[38] J. Huang, H. Xian, and M. Bacaner, Long-Chain Fatty Acids Activate Calcium Channels in Ventricular Myocytes, Proc. Natl. Acad. Sci. U.S.A. 89, 6452 (1992).

[39] D. Schwartzman, R. Bazaz, and J. Nosbisch, Common Left Pulmonary Vein, J. Cardiovasc. Electrophysiol. 15, 560 (2004).

[40] Z. Qu, G. Hu, A. Garfinkel, and J. N. Weiss, Nonlinear and Stochastic Dynamics in the Heart, Phys. Rep. 543, 61 (2014).

[41] J. P. Keener, Model for the Onset of Fibrillation Following Coronary Artery Occlusion, J. Cardiovasc. Electrophysiol. 14, 1225 (2003).

[42] B. E. Peercy and J. P. Keener, Coupled Cell Model of Border Zone Arrhythmias, SIAM J. Appl. Dyn. Syst. 4, 679 (2005).

[43] V. Jacquemet, Pacemaker Activity Resulting from the Coupling with Nonexcitable Cells, Phys. Rev. E 74, 011908 (2006).
[44] K. J. Bishop and B. A. Grzybowski, Localized Chemical Wave Emission and Mode Switching in a Patterned Excitable Medium, Phys. Rev. Lett. 97, 128702 (2006).

[45] I. N. Motoike, S. Nakata, Y. Iguchi, K. K. Takemura, K. Hayashi, and K. Yoshikawa, Apex of a V-Shaped Cut Field Acts as a Pacemaker on an Oscillatory System, Chem. Phys. Lett. 490, 238 (2010).

[46] D. Mihalache, D. Mazilu, F. Lederer, and Y. S. Kivshar, Spatiotemporal Surface Solitons in Two-Dimensional Photonic Lattices, Opt. Lett. 32, 3173 (2007).

[47] S. Batz and U. Peschel, Solitons in Curved Space of Constant Curvature, Phys. Rev. A 81, 053806 (2010).

[48] C. Conti, Localization, and Shock Waves in Curved Manifolds, Science bulletin 61, 570 (2016).

[49] Y. Gaididei, V.P. Kravchuk, and D. D. Sheka, Curvature Effects in Thin Magnetic Shells, Phys. Rev. Lett. 112, 257203 (2014).

[50] H. T. Jadallah, The Onset of Superconductivity in a Domain with a Corner, J. Math. Phys. (N.Y.) 42, 4101 (2001).

[51] P. Bauman, D. Phillips, and Q. Tang, Stable Nucleation for the Ginzburg-Landau System with an Applied Magnetic Field, Arch. Ration. Mech. Anal. 142, 1 (1998).

[52] M. J. Bowick and L. Giomi, Two-Dimensional Matter: Order, Curvature and Defects, Adv. Phys. 58, 449 (2009).

[53] P. Duclos and P. Exner, Curvature-Induced Bound States in Quantum Waveguides in Two and Three Dimensions, Rev. Math. Phys. 07, 73 (1995).

[54] R. DaCosta, Quantum Mechanics of a Constrained Particle, Phys. Rev. A 23, 1982 (1981).

[55] M. Nagasawa, Schrödinger Equations and Diffusion Theory (Birkhäuser, Basel, 2012), Vol. 86.

[56] L.S. Schulman, Techniques and Applications of Path Integration (Courier Corporation, Mineola, 2012).

[57] T. O'Hara, L. Virág, A. Varró, and Y. Rudy, Simulation of the Undiseased Human Cardiac Ventricular Action Potential: Model Formulation and Experimental Validation, PLoS Comput. Biol. 7, e1002061 (2011).

[58] E. Grandi, F. S. Pasqualini, and D. M. Bers, A Novel Computational Model of the Human Ventricular Action Potential and Ca Transient, J. Mol. Cell. Cardiol. 48, 112 (2010).

[59] C. C. Mitchell and D. G. Schaeffer, A Two-Current Model for the Dynamics of Cardiac Membrane, Bull. Math. Biol. 65, 767 (2003).

[60] C. Corrado and S. A. Niederer, A Two-Variable Model Robust to Pacemaker Behaviour for the Dynamics of the Cardiac Action Potential, Math. Biosci. 281, 46 (2016).

[61] https://imagej.nih.gov/ij/.

[62] B. Trenor, K. Cardona, J. F. Gomez, S. Rajamani, J. M. Ferrero, Jr., L. Belardinelli, and J. Saiz, Simulation and Mechanistic Investigation of the Arrhythmogenic Role of the Late Sodium Current in Human Heart Failure, PLoS One 7, e32659 (2012). 